\title{
Smart Walker Solutions for Physical Rehabilitation
}

\author{
Octavian Postolache, José Miguel Dias Pereira, Vítor Viegas, Luisa Pedro, \\ Pedro Silva Girão, Raul Oliveira, and Gabriela Postolache
}

I $\mathrm{n}$ the last decade, the clinical reasoning in physical therapy has been to develop systems for physiotherapists to make clinical decisions rapidly, effectively and efficiently, in response to the increasingly complex needs of health and rehabilitation units [1]-[3]. Some studies show the importance of walking aids during rehabilitation from some diseases, and after surgery for arthroplasty in the elderly population [4], and in elderly patients with balance disorders, muscle weakness [5] or in people with diabetes mellitus [6]. Walkers are important devices that aid the rehabilitation process. The use of a walker is recommended for gait changes and imbalance due to various factors, such as surgery of the lower limbs or neurodegenerative changes, especially in the early recovery period [7].

\section{Aging, Walkers, and Physiotherapy}

To improve the quality of life of people affected by motor limitations as they age, a challenging task is to develop new architectures like instrumented smart walkers using software that assist users increase their balance, and diminish falling [8]-[10], which are major causes of morbidity for the elderly [11]. At the same time, the use of smart equipment with communication capabilities and software associated with electronic health records [12] will allow objective evaluation of physical rehabilitation programs. Various research groups and industries are developing new devices that help users stay balanced by giving a wide base of support.

Types of walkers include the standard walker with no wheels, the two wheeled walker, and the four wheeled walker. The choice of one of these types of walkers is related to the user's gait limitations and stability requirements. Standard and two wheeled walkers are generally used as primary walking aids. General use during rehabilitation proves to improve confidence and restore or maintain motor ability at the highest possible level. A study in [13] showed that walker assistance does not interfere with rehabilitation outcome and in some cases may decrease the rehabilitation period. The study was based on subjective observations of walker users.

\section{Physical Rehabilitation Assessment Network}

To permit continuous monitoring for the physical rehabilitation field, walking aids need to have sensors to measure the parameters related to walking, data acquision and processing, communication units, and network capabilities for remote assessment of the users. A physical rehabilitation network architecture, presented in Fig 1., would include a set of smart walker nodes with wireless communication protocols. We considered Wi-Fi, Bluetooth, Bluetooth LE and IEEE802.15.4 for use because of the required communication ranges, data flow, communication security concerns and the need for autonomy.

The Physical Rehabilitation Network we developed uses Wi-Fi mobile nodes and transmits data from home or clinicbased clients to physiotherapists using an Internet connection. As part of client-server architecture, the data coming from clients is stored in a remote database installed in a Physiotherapy Server where it can be accessed by different web or mobile applications associated with remote physiotherapy to evaluate the rehabilitation sessions in an objective manner. To monitor the walker's activity during gait rehabilitation training, the walker's sensors could measure forces, acceleration, and lower limb motion patterns. Considering the health condition of the users the measurement of cardio-respiratory parameters such as heart rate, and saturation of peripheral oxygen (SPO2) may also help the physiotherapist to optimize the rehabilitation sessions. Research work related to cardio-respiratory assessment through smart walker is reported in [14].

\section{Instrumented Walkers - Smart Walkers}

The measurement of quantities such as forces, accelerations, velocities, heart rate, and oxygen saturation during

This work was supported by the Instituto de Telecomunicações and Fundação para a Ciência e Tecnologia and the project “EHR-Physio: Electronic Health Records-Needs, Requirements and Barriers in Physiotherapy", PTD/DTP-DFS/1661/2012. 
a training session is performed through the use of sensors and signal conditioning circuits embedded on the walker level. Analog processing and signaling circuits may provide visual information to physiotherapists regarding the user's balance and risk of falling. However, advanced features such as gait rehabilitation metrics calculations, gait pattern recognition, power management, and selective communication with the physiotherapy clients requires added acquisition, digital processing and communication modules that transform the instrumented walkers into smart

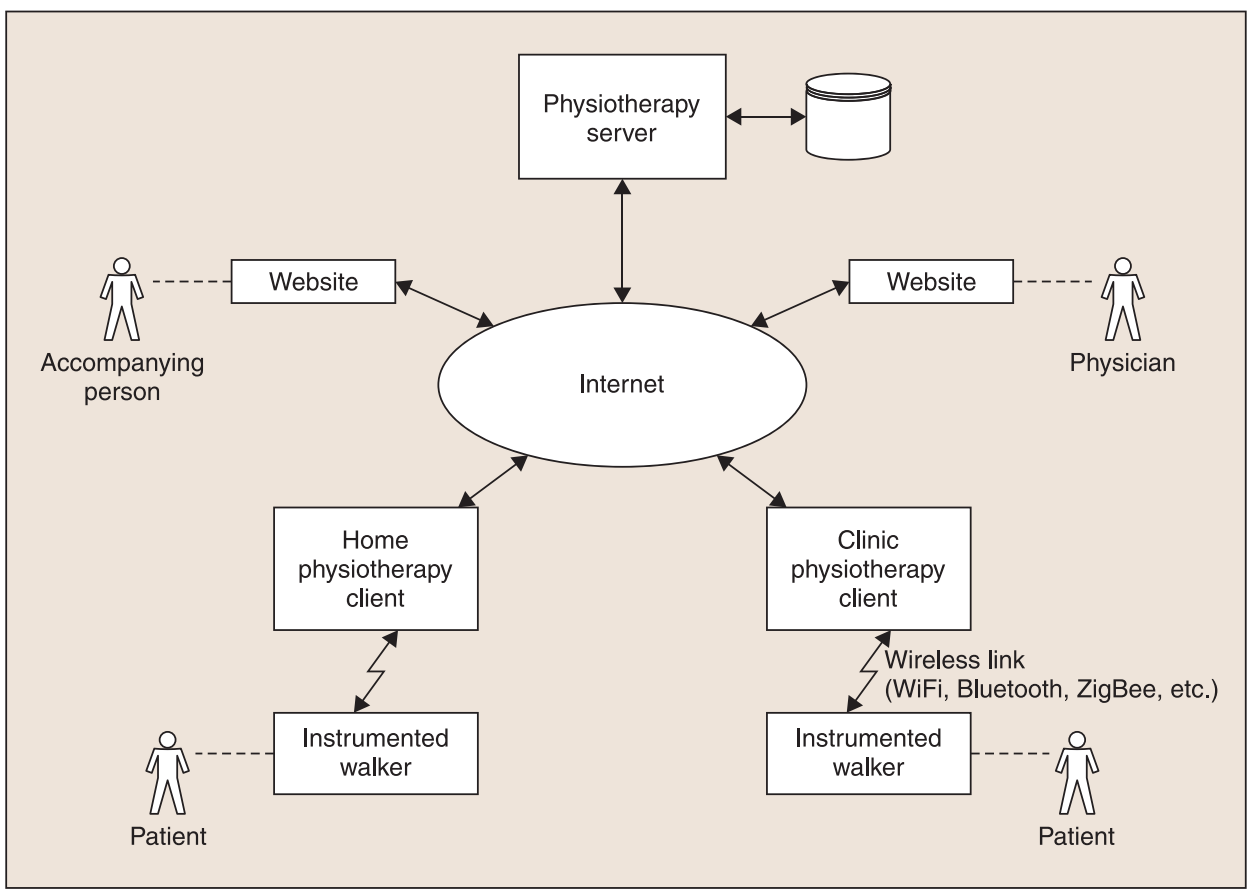

Fig. 1. Physical Rehabilitation Assessment Network based on Wi-Fi smart walkers. walkers. Smart walker

prototypes associated with the Physical Rehabilitation Assessment Network are presented in Fig. 2. The prototypes are characterized by one or two motion sensors (rad, rad1, rad2) that measure the force and acceleration during the gait rehabilitation session (standard smart walker, $2 \mathrm{~W}$ smart walker) or during common daily activity ( $4 \mathrm{~W}$ smart walker).

\section{Smart Walker Sensors}

The sensors embedded in the walker were chosen to extract the relevant information related to the walker's use during the physiotherapy sessions such as applied forces, accelerations and motion patterns.

\section{Force versus Pressure Sensors}

The relationship between force and pressure is given by:

$$
p=\frac{F}{S}
$$

where $F$ represents the applied force, $S$ the surface area and $p$ represents the pressure. The force distribution over a surface is not uniform and the pressure is not constant. There are some common applications where the measurement of pressure distribution over a support surface of a mechanical structure is essential to avoid excessive loading that cause their collapse. In the case of biomedical applications, for example, gait parameters from patients undergoing physiotherapy treatments can be extracted by having the patient use smart insoles equipped with force or pressure sensors to measure ground reaction forces distribution during locomotion with or without aiding walking devices [15]. For walker solutions that can be used for rehabilitation purposes, interface pressure sensing technologies based on load cells, pressure-mapping systems, and force sensing resistors (FSR) will be the most promising sensing technologies in this field.

\section{Force and Pressure Sensing Technologies}

Load cells sensing is based in the deformation of a material when it is submitted to a mechanical effort. For small deformations, the relationship between stress and applied load is given by:

$$
\sigma=\frac{\mathrm{F}}{\mathrm{A}}
$$

where $\sigma$ represents the normal stress, $F$ represents the load and $A$ represents the cross sectional area. Assuming an elastic behavior of the material, the normal stress and the axial or transversal strain are given by:

$$
\begin{aligned}
\varepsilon_{L} & =\frac{\sigma}{Y} \\
\varepsilon_{\mathrm{T}} & =-v \cdot \frac{\sigma}{Y}
\end{aligned}
$$

where $\varepsilon_{L}$ and $\varepsilon_{\mathrm{T}}$ represent the longitudinal and transversal strains, respectively, $v$ represents the Poisson ratio of the material, and $Y$ represents its Young modulus.

Usually, resistive strain gauges are used to sense the deformation and the signal conditioning circuit that is associated with these kinds of sensors is based on Wheatstone bridges. Since the resistance variations are very low, sometimes on the order of a few tens of $m \Omega$, full configuration of the bridge circuit with four sensors is used to compensate errors caused by temperature variation and to increase measurement sensitivity. The load cells work as force sensors since, by themselves, they can only give information about the total force applied to its bearing structure and cannot give information about the 
Based on the information provided by the sensors, individual training sessions can be selected by the physiotherapist to extract information about the evolution of physical capabilities of the trained walker's user.

\section{Conclusions}

Smart walkers represent an important research field as healthcare needs increase with aging. Implementing physical rehabilitation assessment networks based on smart walker nodes that measure force, acceleration and motion allows remote monitoring of physical rehabilitation sessions using Internet connectivity and mobile applications. The applications may also allow sensor data storage and analysis based on client-server architecture. The results that were obtained showed clearly that the proposed technologies can give important contributions to improve the quality of life of walker users by monitoring unbalance and instability conditions that can result in falls and harmful injuries.

\section{Acknowledgment}

The authors give special acknowledgment to MSc. Ruben Costa who collaborated with the project team on mobile applications development.

\section{References}

[1] I. Edwards, M. Jones, J. Carr, A. Braunack-Mayer and G. M. Jensen. "Clinical reasoning strategies in physical therapy," Phys. Ther., vol. 84, pp. 312-330, 2004.

[2] P. M. Masley, C.-L. Havrilko, M. R. Mahnensmith, M. Aubert and D. U. Jette, "Physical therapist practice in the acute care setting: a qualitative study," Phys. Ther., vol. 91, pp. 906-919, 2011.

[3] B. A. Smith, C. J. Fields, and N. Fernandez, "Physical therapists make accurate and appropriate discharge recommendations for patients who are acutely ill," Phys. Ther., vol. 90, pp. 693-703, 2010.

[4] J. A. Singh and D. G. Lewallen, "Predictors of activity limitation and dependence on walking aids after primary total hip arthroplasty," J. Am. Geriatrics Soc., vol. 58, pp. 2387-2393, 2010.

[5] E. Cetin, J. Muzembo, V. Pardessus, F. Puisieux, and A. Thevenon, "Impact of different types of walking aids on the physiological energy cost during gait for elderly individuals with several pathologies and dependent on a technical aid for walking," Annals of Physical and Rehabilitation Medicine, vol. 53, pp. 399-405, 2010.

[6] T. R. de Mettelinge, D. Cambier, P. Calders, N. Van Den Noortgate, and K.Delbaere, "Understanding the relationship between type 2 diabetes mellitus and falls in older adults: a prospective cohort study," PLOS ONE, vol. 3, no. 6, e67055, Jun. 2013.

[7] S. Stowe, J. Hopes, and G. Mulley. “Gerotechnology series: 2. walking aids," European Geriatric Medicine, vol. 1, pp. 122-127, 2010.

[8] O. Postolache, P. Girão, J. M. Dias Pereira, and G. Postolache; "Smart walker for pervasive healthcare," in Proc. Int. Conf. on Sensing Technology - ICST, Palmerston North, New Zealand, pp. 1-5, Dec. 2011.

[9] G. Postolache, P. M. Girão, and O. Postolache, "Applying smartphone apps to drive greater patient engagement and personalized physiotherapy," in Proc. IEEE Int. Workshop on Medical Meas. and Applications 2014, Lisbon, Portugal, pp. 1-6, Jun. 2014.

[10] V. S. Probst, "Mechanisms of improvement in exercise capacity using a rollator in patients with COPD," Chest, vol.126, no.4, pp.1102-1107, 2004.

[11] J. M. Housdorf, D. A. Rios, and H. K. Edelber, "Gait variability and fall risk in community living older adults: an 11-year prospective study," Archives Phys. Med. Rehab., vol 82, no. 8, pp. 1050-1056, 2001.

[12] J. Linder, J. Ma, D. Bates, B. Middleton, and R. Stafford, "Electronic health record use and the quality of ambulatory care in US," [Online]. Available: http:/ / archinte.jamanetwork.com/ article.aspx?articleid $=412773 \&$ resultClick $=1$.

[13] L. Vogt, K. Lucki, M. Bach, and W. Banzer, "Rollator use and functional outcome of geriatric rehabilitation," J. Rehabilitation Res. Dev., vol. 47, no. 2, pp. 151-156, 2010.

[14] A. D. C. Chan and J. R. Green, "Smart rollator prototype," in Proc. IEEE International Workshop on Medical Measurements and Applications, 2008. pp. 97-100, 2008.

[15] S. M. Bradley and C. R. Hernandez, "Geriatric assistive devices," Am. Fam. Physician, vol. 84, no. 4, pp. 405-411, 2011.

[16] J. M. Dias Pereira, O. Postolache, V. Viegas, and P. S. Girão, “A low cost measurement system to extract kinematic parameters from walker devices," in Proc. Int. Instrum.Meas. Tech. Conf. 2015, I2MTC 2015, Pisa, Italy, May 2015.

[17] "Force sensing resistors: integration guide and evaluation parts catalog," Interlink Electronics, (Sep. 2014), [Online]. Available: http:/ / www.interlinkelectronics.com.

[18] “WalkwayTM gait analysis system", Tekscan, (Jun 2015), [Online]. Available at: https://www.tekscan.com/sites/default/ files/resources/MDL-Walkway-System-Datasheet.pdf.

[19] K. Lauszus, (2012) “A practical approach to Kalman filter and how to implement it," [Online]. Available: http://blog.tkjelectronics. dk/2012/09/a-practical-approach-to-kalman-filter-and-how-toimplement-it/.

[20] "Kalman filter vs. complementary filter," Robottini, [Online]. Available: http:/ / robottini.altervista.org/kalman-filtervscomplementary-filter.

[21] O. Postolache, P. S. Girão, G. Postolache, and G. Mendes. "Cardio-respiratory and daily activity monitor based on FMCW Doppler radar embedded in a wheelchair," in Proc.33rd Annual Int. Conf. IEEE Eng. in Medicine and Biology Soc., pp. 1917-1920, 2011.

[22] O. Boric-Lubecke, V. M. Lubecke, I. Mostafanezhad, B.-K. Park, W. Massagram, and B. Jokanovic," Doppler radar architectures and signal processing for heart rate extraction," Mikrotalasna Revija, pp. 12-17, Dec. 2009.

[23] T. Yardibi, P. Cudihy, S. Genc, C. Bufi, M. Skubic, M. Rantz, L. Liu, and C. Phillips II, "Gait characterization via pulse-doppler radar," in Proc. Smart Environments to Enhance Healthcare, pp. 662-667, 2011.

[24] O. Postolache, M. D. Pereira, V. Viegas, and P. Girão,"Gait rehabilitation based on microwave Doppler radars embedded in walkers", in Proc. IEEE Int. Workshop on Medical Meas. and Applications, MeMeA 2015, Torino, Italy, pp. 1-6, 2015. 
Octavian Adrian Postolache (octavian.postolache@gmail. com) (M'99, SM'2006) graduated in Electrical Engineering at the Gh. Asachi Technical University of Iasi, Romania, in 1992 and he received the Ph.D. degree in 1999 from the same university. He is Senior Researcher of Instituto de Telecomunicações and, since 2012, he joined ISCTE-IUL Lisbon where he is currently assistant professor and IT-IUL member of scientific council. His fields of interests are smart sensors for biomedical and environmental applications, pervasive sensing and computing, wireless sensor networks, and computational intelligence applied in instrumentation. Dr. Postolache is author and co-author of nine patents, seven books, fourteen book chapters, 65 papers in international journals with peer review, and more than 200 papers in proceedings of international conferences.

J. M. Dias Pereira (M'00-SM'04) received his degrees in Electrical Engineering from the Instituto Superior Técnico (IST) of the Technical University of Lisbon (UTL). For almost eight years, he worked for Portugal Telecom in digital switching and transmission systems. In 1992, he returned to teaching as an Invited Professor in Escola Superior de Tecnologia of Instituto Politécnico de Setúbal where he is a Principal Coordinator Professor. His main research areas include smart sensing, data sensor fusion, A/D and D/A conversion techniques, instrumentation and transducers for industrial applications, and advanced data processing techniques for biomedical and environmental applications.

Vitor Viegas received his degree in Electrical \& Computer Engineering from the Instituto Superior Técnico/Universidade de Lisboa in 1999, followed by the M.Sc. and Ph.D. degrees in 2003 and 2012, respectively. He works as Assistant Professor at the Polytechnic Institute of Setúbal (www.ips.pt), and as a Researcher at the Instituto de Telecomunicações (www.it.pt). His main research activities concern smart transducers, industrial informatics and biomedical instrumentation.

Luísa Maria Reis Pedro obtained the M.Sc. and Ph.D. degrees from the Higher Institute of Applied Psychology and University of Porto Faculty: Faculty of Psychology and Educational Sciences in 2003 and 2007. She is an assistant professor at the Institute Polytechnic of Lisbon - College of Health Technology of Lisbon. Her research interests involve intervention programmer for the promotion of autonomy, quality of life, and satisfaction with life in individuals with multiple sclerosis. She has published about 30 articles in national and international proceedings and scientific journals with referee, and she has made about 70 oral and poster presentations in national and international conferences.

Pedro Silva Girão $\left(\mathrm{M}^{\prime} 00-\mathrm{SM}^{\prime} 01\right)$ is a Full Professor of the Department of Electrical Engineering, Instituto Superior Técnico, University of Lisbon, and a Senior Researcher, the Head of the Instrumentation and Measurements Group, and the Coordinator of the Basic Sciences and Enabling Technologies of the Instituto de Telecomunicações. His main research interests include instrumentation, transducers, measurement techniques, and digital data processing, particularly for biomedical and environmental applications. Metrology, quality, and electromagnetic compatibility are also areas of regular activity. Dr. Girão is a Senior Member of the IEEE.

Raul Oliveira received his M.Sc. in Child Development and Ph.D. in the Motricity Sciences. He received both degrees from the Faculdade de Motricidade Humana, Universiadde de Lisboa in 1999 and 2010 where he is currently an assistant professor. He is a researcher for the Laboratory of Motor Behavior and an expert reviewer for the Swiss National Science Foundation (SNSF) as reviewer of important national and international scientific journals. His research interests are related to neuromuscular adaptations to training, sports, and physical therapy. He is the author or co-author of nine books and articles published in national and international physiotherapy science journals and he is member of the project team "Electronic Health Record for Physiotherapy."

Gabriela Postolache is researcher at Unidade de Fisiologia e Clinica Translacional, Instituto de Medicina Molecular, Universidade de Lisboa. She obtained a Ph.D. degree in Life Sciences in 2006. Her research interests include electrophysiology, biomedical instruments, non-invasive and unobtrusive measurements of body signals. She was involved in research projects related with autonomic nervous system assessment and monitoring, cardiac arrhythmia, electronic health records for wheelchair users, electronic health records for physiotherapy and she is author and co-author of several patents. 